\title{
An Anti-collision Structure for Bridge Piers Made by Waste Container
}

\author{
LIU Jin-long ${ }^{1, a}$, LIU Jie-qun ${ }^{2, b, *}$ and ZHU Lei ${ }^{1, c}$ \\ ${ }^{1}$ Department of Civil Engineering, Hefei University, Hefei 230601, China \\ ${ }^{2}$ Department of Computer Science and Technology, Hefei University, Hefei 230601, China \\ aalnile@163.com, 'ljq109p@163.com, 523260684@qq.com \\ *corresponding author
}

\begin{abstract}
An anti-collision structure for bridge piers made by waste containers is proposed in this article, which including waste containers and filling. The top of container is cut and removed, a number of containers arranged with different styles on the main route of rolling stones and in front of the bridge piers to be protected. Rock and soil is filled in the cavity of container. The energy generated by rolling stones acts on the used containers instead of acting directly on the bridge piers. It is economical, convenient and fast construction speed to build the anti-collision structure with waste containers around bridge piers.
\end{abstract}

Keywords: bridge pier; rolling stone; collision; waste container

\section{Introduction}

Bridge piers are the key parts and weak links in the bridge construction, which need to be protected during the traffic engineering operation. Bridges located in high mountain canyons are often threatened by various geological disasters, including collapsing rock[1, 2], debris flow, avalanches, etc. These geological disasters may cause damage and destruction to piers.

For example, on July 25, 2009, large-area collapse occurred in the high rock mass on the right bank of the Minjiang River of China[3,4], the total volume of the collapsed mass exceeded 10,000 cubic meters. The 8 \# bridge pier of Chediguang bridge, located below the location of the collapse, was instantly broken by boulders rolling down along steep slopes, resulting in the 8th and 9th beam body of bridge a total length of $60 \mathrm{~m}$ falling, and the maximum offset of the 10th and 11th beam body of bridge arrived $26 \mathrm{~cm}$, the expansion joints of the 11th bridge abutment damaged. During this accident, 6 people died, 12 people injured, and 7 vehicles were damaged by the rockfall disaster, as shown in Figure 1.

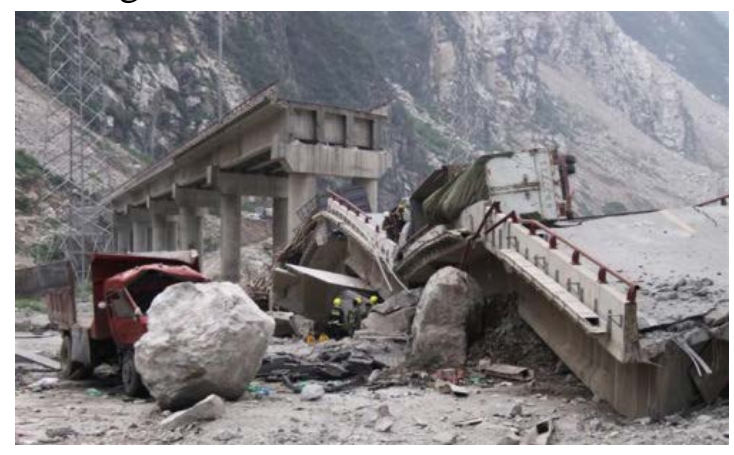

(a) Collapse of bridge piers

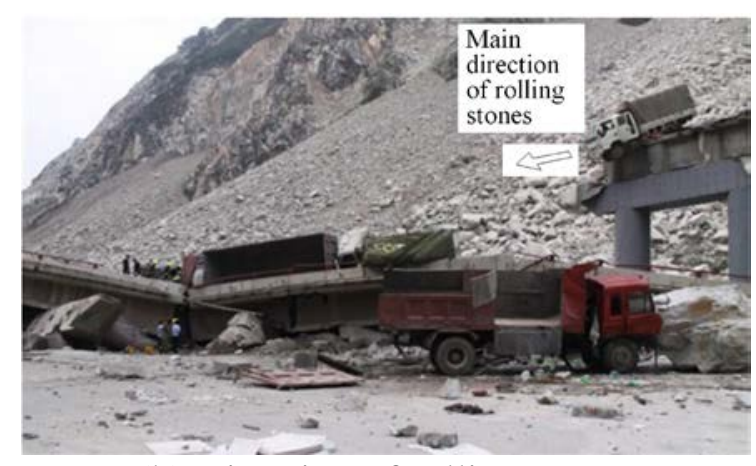

(b) Direction of rolling stones

Fig. 1 Chediguang bridge of China was damaged by rolling stones

In the current technology, the anti-collision structure for bridge piers is often installed on the external face of bridge piers. Usually, the anti-collision structure is composed of a plurality of protection units 
which are arranged on the outer wall of bridge pier from top to bottom, each protection unit is composed of a plurality of protection components, which comprises an inner structure filled with filler and an outer structure composed of stiffeners and separated into a closed cavity[5 8]. While this kind of anti-collision structure installed, fixed around the outer wall of piers, the impact energy of the rolling stone is finally passed on to the pier through anti-collision structure, the role of anti-collision structure is only to reduce the peak load impact strength, which did not reduce the total impact energy. So, it is necessary to develop another kind of simple and practical bridge pier anti-collision structure.

\section{Anti-collision structure made by waste container}

China is a big country in the production and use of containers, $94 \%$ of the containers in the world are manufactured in China. At the same time, more than 300,000 used containers have been eliminated each year in China, as shown in Figure 2. Redevelopment of used containers is expensive, and it is waste and poor environmental protection for waste container recycling steelmaking. At present, only a small amount of containers have been transformed into houses, temporary housing construction and mobile exhibition halls. Therefore, there is still a need to rebuild and utilize a large quantity of used containers to find out more potential value.

Container is a kind of equipment which is made of steel for the transportation of goods. The main components include top beam, bottom beam, door frame, corner column, corner piece, etc, with the character of strong, solid structure, high weldability, and good water tightness. If used container can be used to make anti-collision structure of bridge pier, it can reduce the cost and construction difficulty of anti-collision structure, and also provide a new way for reusing the used containers, which is apparently in line with the development of resource recycling demand.

This paper intends to provide an anti-collision structure of bridge pier made by waste container, mainly consists of waste containers and filling. The top of container should be removed, as showed in Fig. 3. The illustration tags in this article are uniformly described as follows: 1 - used container; 2--corner piece; 3-reinforced concrete pile cap; 4-bridge pier; 5-- filling; 6--masonry.

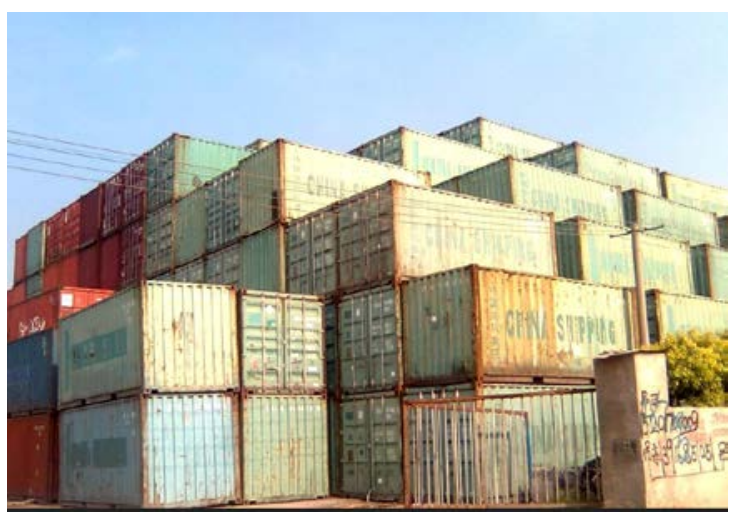

Fig. 2 Stacks of used containers

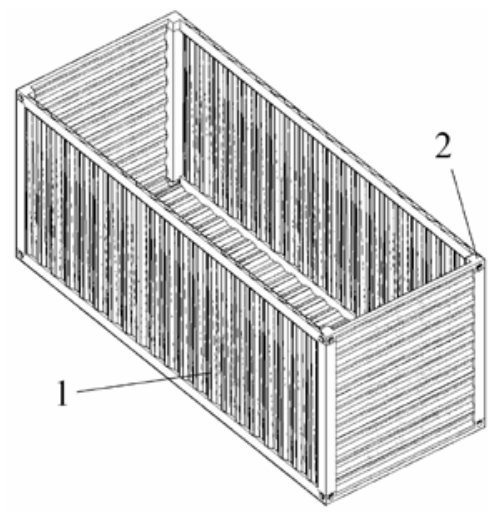

Fig. 3 Diagram of container removed the

top

When only a waste container is used, the container could be placed on ahead of the bridge piers to be protected to resist rolling stones. The long sides of the container perpendicular to the main direction of rolling stones, and the cavity of the container is filled with filling, as shown in Fig. 4 and Fig. 5.

Take the container with the specification of 20GP as an example, the external dimensions are $6.058 \mathrm{~m} \times 2.438 \mathrm{~m} \times 2.591 \mathrm{~m}$, the cavity volume is $33.1 \mathrm{~m}^{3}$ and the tare weight is $2.3 \mathrm{t}$. Assuming that the density of the filling is $1700 \mathrm{~kg} / \mathrm{m}^{3}$, so the weight of filling of each container is $33.1 \times 1700=56270 \mathrm{~kg}=56.2 \mathrm{t}$, and the total weight of individual containers (including tare weight) can reach 58t. The total weight of container with filling is very larger, so the overall stability of container is very good, which can withstand greater impact energy of rolling stones. 


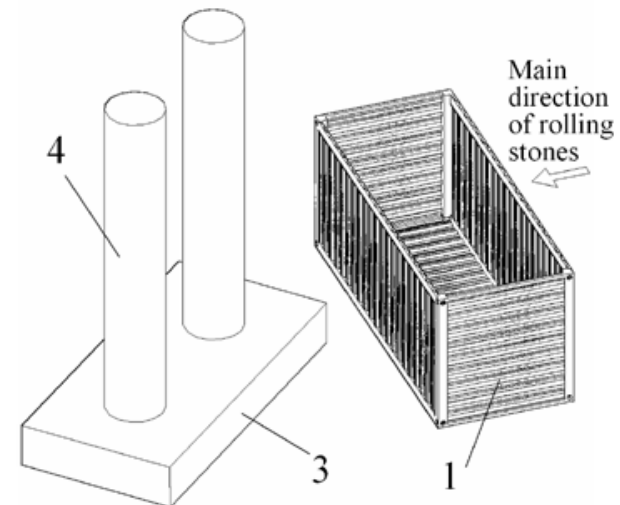

Fig. 4 Diagram of using a single container

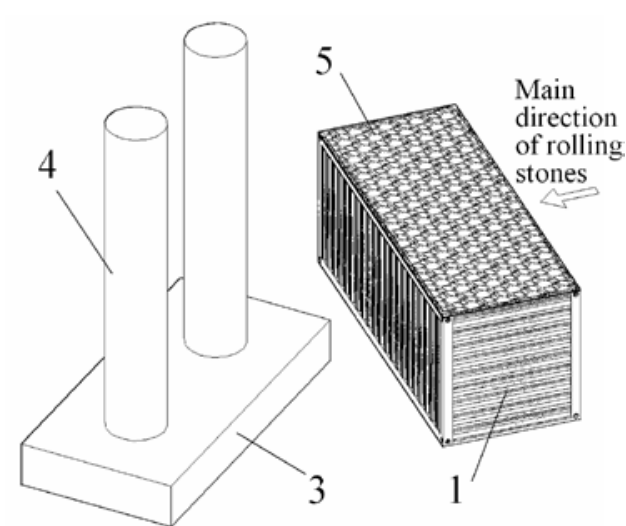

Fig.5 Diagram of filled with filling

There is a certain distance between anti-collision structure and bridge piers, so the rolling stones first collide with the anti-collision structure without acting on the bridge pier. That is, the energy generated by the rolling stones acts on the waste container with filling instead of acting directly on bridge pier. Only when the anti-collision structure is damaged by impact, the remaining energy was transmitted to bridge pier. Compared with the traditional anti-collision structure installed and fixed around the outer wall of bridge pier, the proposed device can greatly improve the protection effect.

The bottom of the anti-collision structure does not press on the top of reinforced concrete pile cap, that is, the anti-collision structure will not cause additional settlement of the bridge pier. Containers should be treated with antirust treatment before use, and multi-row holes drilled symmetrically on the floor of container for water discharge in the filling materials.

The filling is rock and soil dug near the bridge pier. A thin layer of sand or medium sand with a thickness of not less than $15 \mathrm{~cm}$ shall be laid on the floor of the container, so that water in the filling can be drained from the holes in the floor of the container.

When two same containers are used, the side walls of the two containers could be aligned and contact with each other, and the adjacent corner fittings are fixedly connected by the fixing inserts, as shown in Fig. 6 . The combined containers are placed on the main route of rolling stone and on the front of the bridge pier to be protected. The long sides of the container are perpendicular to the main direction of rolling stones. The total weight of two containers (including tare and filling) can reach $116 \mathrm{t}$. If the main route of rolling stone with wide range, the two waste containers can be arranged in series, aligned with the end wall and in close contact with the adjacent corner fittings fixedly connected, as shown in Fig. 7.

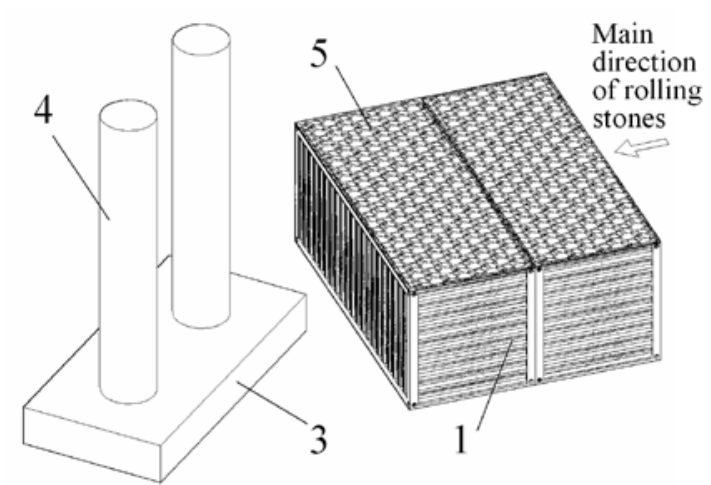

Fig. 6 Two containers side by side

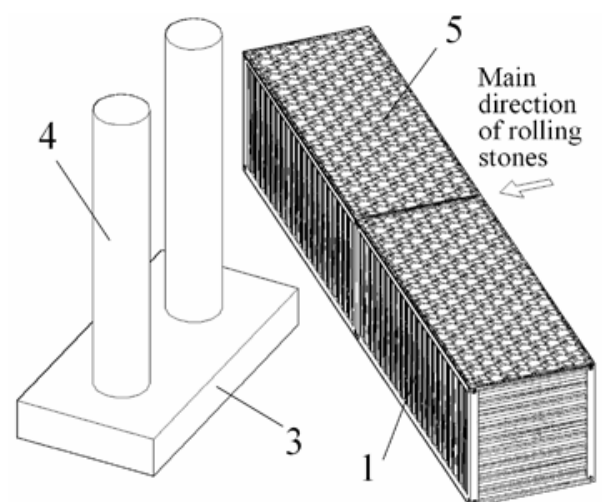

Fig.7 Two containers arranged in series

Three containers can also be used, stacked in a single layer or two layers, as shown in Fig. 8 and Fig. 9. Fig.10 shows a four-container arranged in two layers.

In addition to the straight line arrangement, the containers can also be arranged in polylines, as shown in Figure 11, which shows a case where two containers are tilted arranged. The two waste 
containers are symmetrically arranged on both sides of the main direction along the main route of rolling stones at a certain angle. Two corners of the used containers are in close contact with each other, and the adjacent corner fittings are fixedly connected by a fixed plug. The cavity of each container is filled, and masonry built at the triangular open space formed at the corner of the two container end walls.

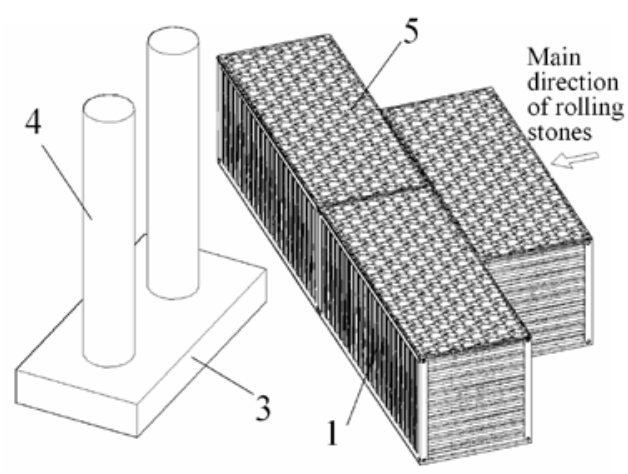

Fig. 8 Three containers arranged in a single layer

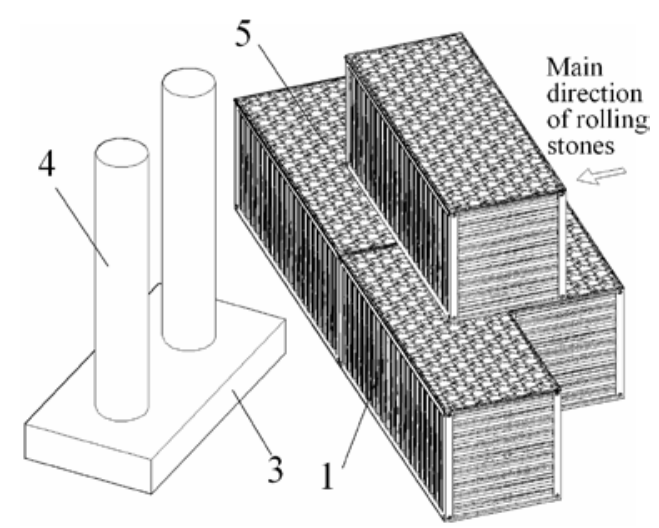

Fig. 10 Four containers arranged in two layers

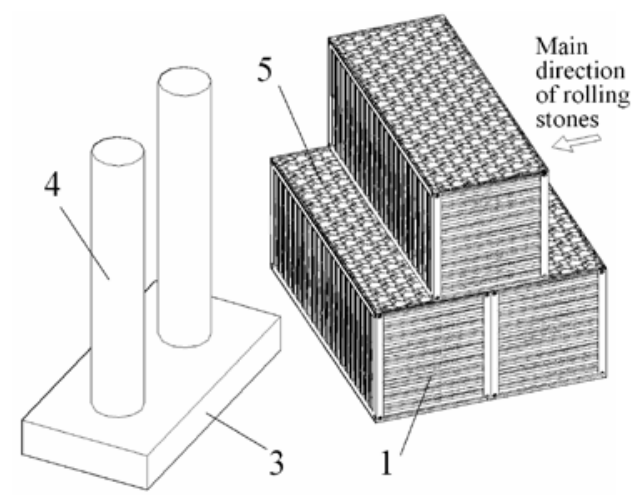

Fig.9 Three containers arranged in two layers

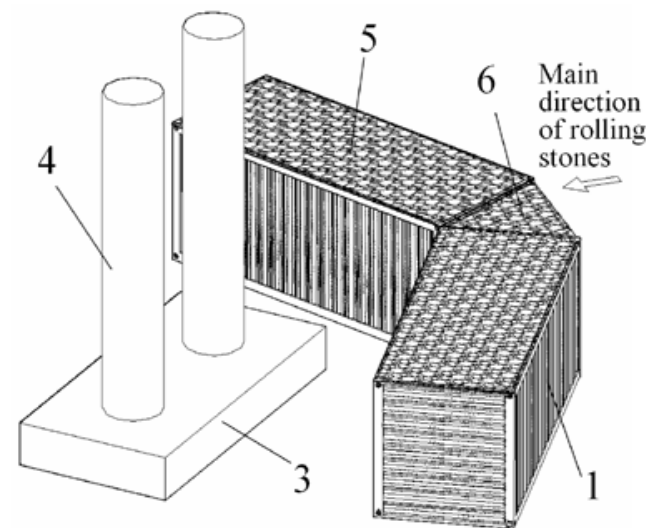

Fig.11 Two containers tilt arranged

The anti-collision structure made by waste containers proposed in this article could be constructed with the following steps:

(1) Cut and remove the top of the used container, as shown in Fig. 3. Multi-row holes should be symmetrically drilled at the bottom of the container evenly, so water could be discharged form filling trough holes.

(2) Determine the location of the container to be placed, where need to be flat and compacted. Usually, containers should be placed on the main route of rolling stones and at the front of bridge pier need to be protected, and containers should not on the top of reinforced concrete pile cap.

(3) Stack containers on the selected position, and a thin layer of sand or medium sand with a thickness not less than $15 \mathrm{~cm}$ shall be laid on the floor of container so that water in the filling can be drained from the holes at the bottom plate of container. Rock and soil is to be thrown into the cavity of the container. Heavy materials such as block stone, gravel, flake and gravel shall be filled in the middle and lower parts of the container, and filling should be layered compacted.

The anti-collision structure made by waste containers proposed in this article has the following advantages:

(1) The anti-collision structure is simply processed by used container, with the characteristics of strong intensity, solid structure and large volume, which provided a new way for resource reuse of the used container. It is simple, convenient and fast construction speed to build the anti-collision structure around bridge piers.

(2) The total weight of container with filling is more than 50t, which can bear huge impact energy of rolling stones. The overall stability of anti-collision structure is very big. 
(3) There is a certain distance between anti-collision structure and bridge piers, so the energy generated by rolling stones acts on the used container instead of acting directly on the pier. Only when the anti-roll structure is damaged by collision, the remaining energy will transmit to the bridge pier. Compared with the traditional anti-impact device installed and fixed around the outer wall of the piers, the device proposed in this article can greatly improve the protection effect.

(4) The filling is the rock and soil dug near the bridge piers, so, it is convenient and economical to do filling work. The drainage holes drilled at the bottom plate of container so that the interior of filling body is always dry, which make the container not easy to rust and with longer service life.

\section{Concluding remarks}

The traditional anti-collision structure is installed and fixed around the outer wall of bridge piers usually, its role is to reduce the peak of impact strength, but did not reduce the total value of impact energy, all the impact energy of rolling stones is finally passed to the bridge piers. In this paper, an anti-collision structure made by waste container is provided, which is placed in front of the bridge piers to be protected. The energy generated by rolling stones acts on the used container instead of acting directly on the pier. Only when the anti-roll structure is damaged by collision, the remaining energy will transmit to the bridge pier, so the device proposed in this article can greatly improve the protection effect.

\section{Acknowledgments}

This work was financially supported by Anhui provincial department of education program on college teachers visiting abroad under grant No. gxfxZD2016216, Anhui province university natural science research project under grant No. KJ2015A151 and Hefei university academic leaders project under grant No. $2016 \mathrm{dtr} 01$.

\section{References}

[1] Shen Jun, He Siming, Wu yong. Present research status and development trend of rockfall hazards, Journal of Catastrophology, 2008, 23(4): 122-125.

[2] Yu Zhixiang, $\mathrm{Xu} \mathrm{Hu}$, Lu Lei, et al. Influence on damage of bridge pier in mountain area under impact load of rockfall, Journal of Sichuang University (Enginerring Science Edition), 2012, 44(6): 86-91.

[3] He Siming, Zhuang Weilin, Zhang Xiong. Research on rockfall impact prevention of Chendiguan bridge pier, Duwhen road, Chinese Journal of Rock Mechanics and Engineering, 2013, 32(Supp. 2): 3421-3427.

[4] Yuan Jinke, Pei Xiangjun. Modeling and study of impact damage to piers of Chediguan bridge, Highway, 2014, 2: 22-26.

[5] He Siming, Wang Dongpo, Wu Yong. Formation mechanism and key prevention technology of rockfalls, Chinese Journal of Nature, 2014, 36(5): 336-345.

[6] YE Xin, Xiong Wen, Zhang Juanxiu. Collision simulation and rigid design between rolling stone and anti-collision structure for bridge piers, Journal of Harbin Institute of Technology, 2015, 47(2): 117-122.

[7] Pu Chao. Test study on impact force of rockfall and the calculation method discuss, Subgrade Engineering, 2015, 6: 110-115.

[8] Yi Wei, Yu Bing, Liu Yang. Research on Calculation Method of Rockfall Impact Force, Mountain Research, 2016, 34(3): 310-316. 